\title{
A DEMONSTRATION RIG FOR CONTROL SYSTEMS BASED ON THE BALL-AND-BEAM WITH VISION FEEDBACK
}

\author{
J. WHET AAN AND J.V. RINGWOOD
}

School of Electronic Engineering, Dublin City University, Glasnevin, Dublin 9, Ireland

\begin{abstract}
Abetract. This paper describes a rig based on the ball-and-beam apparatus which has been used to effectively demonstrate the power of electronic control systems. Though the controller employs some complex electronic systems, its operation closely mimics that of a human performing the same action, and is therefore easily understood. This is achieves through measurement of the ball position and velocity states using a machine vision system.
\end{abstract}

Key. Words. Computer control, educational aids, ball-and-beam, machine vision, PID control

\section{INTRODUCTION}

The ball-and-beam apparatus (Wellstead (1981) represents a popular and easily understandable process to which computer control may be effectively applied. It also has the important characteristic of being openloop unstable, making the application of computer control all the more effective from a demonstration point of view. In addition, the balancing problem associated with the balland-beam is representative of a number of well-known unstable control problems, such as maintaining the vertical position of a rocket taking off, inverted pendulum, etc.

The ball-and-beam apparatus also has the attraction that people with no experience of control systems whatsoever can attempt to control the system using hand movements and compare their control actions (including visual feedback) to those of the computer system. A typical use of the demonstration rig would be along the following lines:

- Invitation to audience to control the system with hand movements, with no computer control,

- An explanation of the components of the computer control system and the way in which they reflect aspects of the human control system, and finally

- demonstration of the operation of the computer control system.
The overall system configuration is shown in Fig.1.

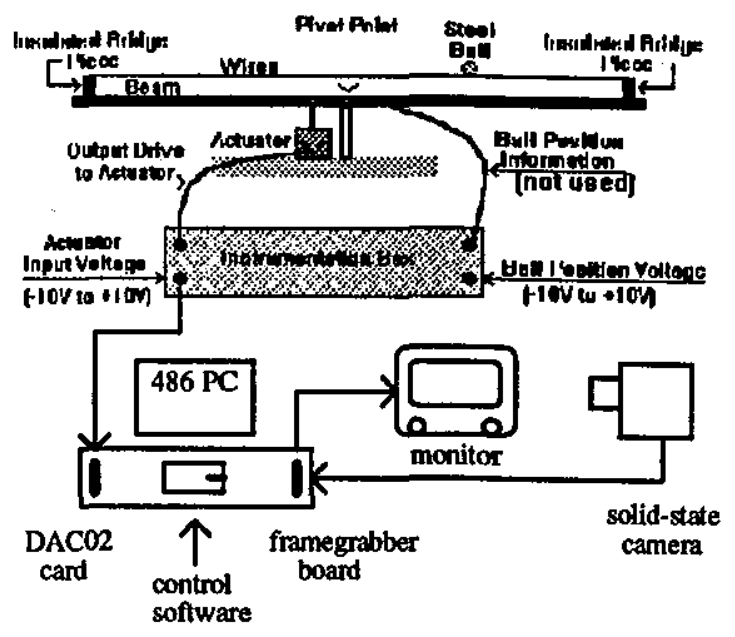

Fig.1 : System Overview

The ball-and-beam is connected, via an instrumentation box, to a $486 \mathrm{PC}$ containing analog output and framegrabber cards. Under more conventional operation, an analog input card would also be provided for (electrical) ball position measurement, but this information is now supplied visually. The PC acceps an image from the solid-state camera, using the framegrabber and relays the image to the monitor, displaying 'what the computer sees'. 


\section{THE BALL-AND-BEAM APPARATUS}

The ball-and-beam is provided as a standard piece of educational apparatus by TecQuipment (Wellstead (1981)) and includes an instrumentation box allowing an electrical position measurement and providing an electrical input for actuation. The ball-andbeam itself consists of an aluminium T-section 1.1 metres long supporting two tautly stretched wires along its length. These wires provide a 'channel' in which a steel ball moves and allow electrical (resistivity) measurement of ball position, if required. The beam is pivoted in the centre and tilted via a linear actuator, attached slightly off beam centre.

The true system is described by a fourth-order nonlinear dynamical equation, but for most purposes a second order linear differential equation suffices, assuming that the beam dynamics are significantly faster than those of the ball, and the beam tilt angle is small. This gives the simplified relation between ball position, $x(t)$ and actuator force $F(t)$ (proportional to the voltage input) as:

$$
\ddot{x}(t)=b F(t)
$$

The parameter $b$ depends on $g$, the acceleration due to gravity and the ball and beam parameters. Note that the double derivative on the output, gives a double pole at $s=0$, indicating the unstable nature of the system.

\section{THE VISION SUBSYSTEM}

The vision subsystem forms the most complex part of the demonstration rig and provides a position measurement by processing of regular samples of the camera image. The components of this subsystem are descirbed in the following subsections.

\subsection{Rig-Modifications}

The rig must be modified to allow efficient processing of the vision information. In particular, to optimise the visual contrast of the system, the ball was painted black, and a white ball background was mounted behind the area of travel of the ball. A grey beam background was also placed behind the apparatus, covering the area of movement of the beam as shown in Fig.2. In addition, two moveable 'targets' were placed on the ball background, allowing visual setpoints to be set.

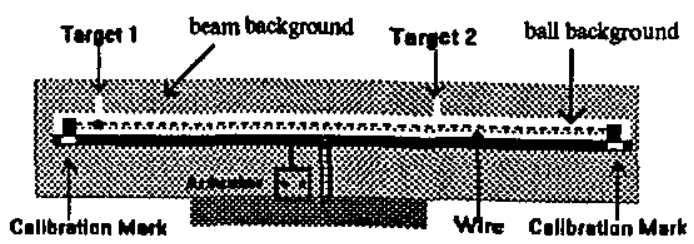

Fig.2. : Modifications to Apparatus

\subsection{The Camera}

The monochrome camera produces a complete scan of the scene every $33.3 \mathrm{~ms}$ (the camera scan rate is $30 \mathrm{~Hz}$ ). This is in interlaced form, and two images are produced, corresponding to even and odd lines. The output of the camera is therefore a burst of continuous signal every $63.55 \mu \mathrm{s}$, which is the chrominance signal for each line followed by a line sync pulse, for a total of 525 lines, including both odd and even lines.

\subsection{The Framegrabber Board}

This board, which is driven by a crystal controlled clock operating at $7.3 \mathrm{MHz}$, samples the chrominance signal (excluding the sync. information) to give 387 pixels per line over 488 lines. Note that only 488 lineas contain chrominance (scene) information, the remainder normally reserved for digital information transmission. The $A / D$ converter codes each pixel with an 8 bit grey level and this is subsequently thresholded to give a black (255) or white (0) value. The digitized image is stored in video RAM on the frame grabber card in matrix form.

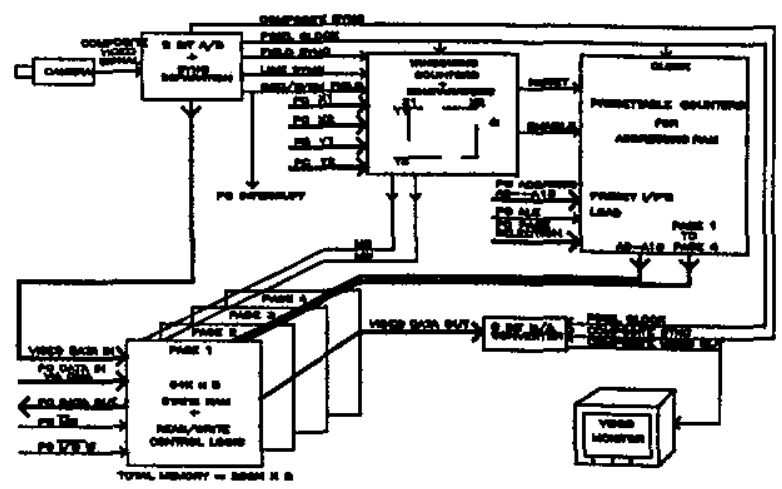

Fig.3. Framegrabber board

Fig. 2 shows the detail of the framegrabber card. The core of this subsystem is the four pages of $64 \mathrm{~K}$ Bytes of static RAM, with chrominance to pixel 
conversion on the camera input and the opposite on the monitor output, together with some counters and read/write/enable logic. An important final feature of the board is a windowing feature, allowing an area of interest (AOI) to be set up, thus avoiding the capture of redundant information. The framegrabber board was designed and built in-house.

\subsection{The Monitor}

The monitor displays a picture of the AOI as the computer sees it. This is useful both for dubugging of the image system and demonstration purposes. The picture is generated from the video RAM on the framegrabber board with the appropriate sync signals, etc. A typical display is shown in Fig.4.

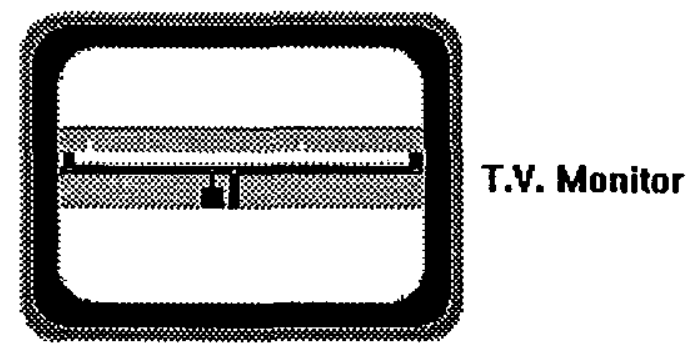

Fig.4. Typical monitor display

\section{IMAGE PROCESSING}

The main objective of the image processing software is the determination of the ball position on the beam, complicated by the fact that the beam is not always horizontal. This requires the beam angle to be determined so that an appropriate rotational transformation may be applied to determine the true horizontal displacement of the ball. Note that when ball is near to balance on the beam, the ball is effectively travelling in the horizontal direction only and capturing and processing the even field image is sufficient, since vertical resolution deminishes in importance. A significant increase in processing efficiency was obtained by dumping the contents of the video RAM on the framegrabber down to $\mathrm{PC}$ RAM. This was due to three factors:

- Memory access time for PC RAM is significantly faster than that for PC EPROM, into which the framegrabber RAM is mapped,

- PC cache memory decreased access to the image data still further, and

- The framegrabber may input another image while the previous image is being processed by the PC.
The following components comprose the image processing suite of functions.

\subsection{Thresholding}

A function was written to allow the average intensity of any number of pixels in a contiguous line to be evaluated. The line of pixels chosen may run at an angle to the horizontal through the rows of pixeis in an image, thereby allowing the average intensity of the ball background to be found.

\subsection{Optimising the Area of Interest Window}

Prior to calibration, an AOI window of 320 pixels wide by 128 lines is set up, approximately matching the length to height ration of the ball-and-beam, including background. The AOI window is displayed on the monitor and the camera moved away from the beam until the outer extremes are just barely visible on the monitor.

\subsection{Slope of Beam}

To find the slope of the beam, the pixels representing the beam are found relative to the top row of pixels in the AOI window. Vertical rows of pixels at the LHS and RHS of the AOI window are processed until the average of a group of three neighbouring pixels has a value of 30 below the average beam background value. The slope is then simply determined from the difference in vertical distances divided by the width of the AOI window.

\subsection{Calibration}

This function brings the beam horizontal, finds the calibration markers on the beam and determines the length of the beam in terms of the number of equivalent pixels. In addition, the positions of the setpoints are determined relative to the centre of the beam. Finally, the optimum tracking line for the ball is found by determining the vertical displacement of the beam.

\subsection{Ball Position}

This function uses the ball tracking line and the average intensity of the ball background in an algorithm that tracks the slope of the beam. This line of pixels is then processed from left to right until the value of three neighbouring pixels has a value of 30 above the background average. 


\section{THE CONTROL SYSTEM}

The control system is based around a digital PID algorithm, chosen primarily for intuitive appeal. Users/onlookers can easily see the change in reponse as different proportions of the pall position or velocity are introduced.

\subsection{Sampling Period Selection}

The sampling frequency must be chosen to satisfy two requirements - fast enough to capture the dynamics of the ball-and-beam, while being slow enough to be implementable. This second factor is determined by the data acquisition time (about $16.6 \mathrm{~ms}$ ) plus the computational time. Computational time is approx. $50 \mathrm{~ms}$ for the determination of the ball position and a further $10 \mathrm{~ms}$ for controller calculations. The resulting maximum sampling frequency on a $33 \mathrm{Mhz} 386 \mathrm{PC}$ is therefore approx. $13 \mathrm{~Hz}$, which is more than adequate, being more than 40 times the closed loop bandwidth (of an equivalent continuous-time system). However, the controller sampling frequency must be synchronised to the camera scan rate of $30 \mathrm{~Hz}$, so a value of $7.5 \mathrm{~Hz}$ is chosen $(=30 / 4)$.

\subsection{PID Controller}

The PID controller is of standard form. Integral control is introduced to improve the response to nonzero setpoints. Anti-integral windup was implemented by resetting the integral to zero following a setpoint change until the rate of change of ball position with time was less than $0.02 \mathrm{~m} / \mathrm{s}$. The controller output is fed to the ball-and-beam via the DAC02 card.

\section{OVERALL SYSTEM OPERATION}

The pipelined operation of the software is demonstrated in Fig.5. This optimises* the computational resources by having simultaneous operation of the PC and framegrabber board. In the controller, for demonstration purposes, a dynamical form of setpoint generation was used. After the system has settled at one setpoint (see Fig.2), as determined by examination of the error and its derivative, the other setpoint is switched in and the process repeated. The response for the system, using the controller settings of $P=12, I=3$ and $D=12$, is shown in Fig.6. The user interface provides the following facilities:

- Display of the AOI on the monitor

- Changing of the setpoints

- Entering the P, I and D settings

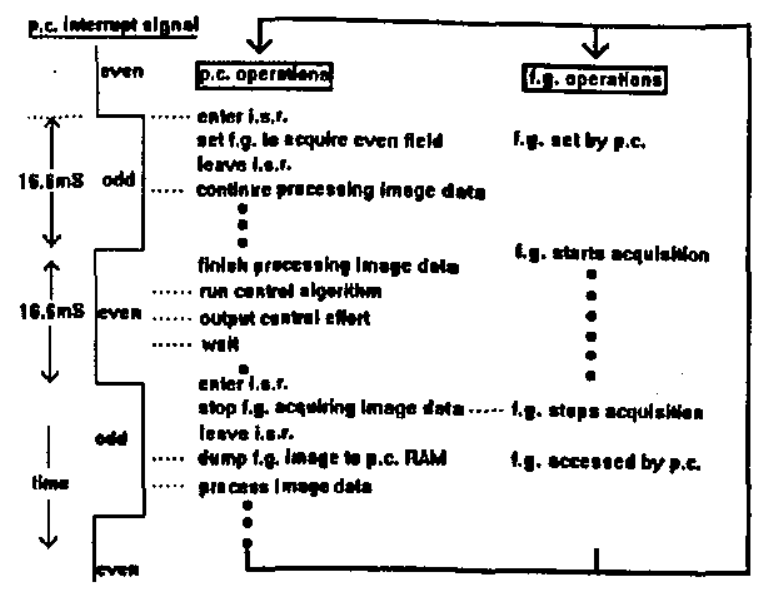

Fig.5. Flowchart Showing Pipelining

During program execution, three options are available:

- Let program run indefinitey

- Change PID settings or setpoints

- Quit program completely

- Stop/start control loop execution

The final option above is implemented using the vision system. If a user wishes to terminate program execution, he/she simply waves a hand in front of the camera. Resumption of program execution is established in the same way.

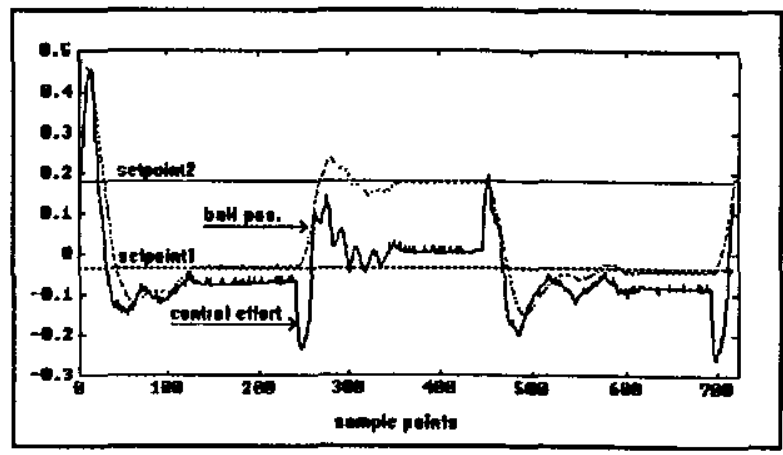

Fig.6. System Dynamic Response

\section{CONCLUSIONS}

The rig has been found to be very effective in demonstrating the use of computer control systems, mainly by virtue of the close parallel with the human system. The vision-based feedback utilises the full potential of the PC, and is immune to sensor noise and drift.

\section{REFERENCES}

Wellsead, P.E. (1981). CE6 - Ball and Beam Apparatus. TecQuipment Ltd. 